$>$ Au cours de l'épidémie de maladie à virus Ebola qui a affecté l'Afrique de l'ouest entre 2013 et 2016, la mise en place d'essais cliniques en vue de l'évaluation de stratégies thérapeutiques et de vaccins prometteurs, mais non homologués, est apparue comme une composante emblématique de la réponse globale à l'épidémie. En 2017, aucun agent antiviral n'a pu prouver une efficacité définitive chez les patients. Deux produits, la combinaison d'anticorps monoclonaux ZMapp et l'antiviral direct favipiravir, testés au cours d'essais cliniques de preuve de concept ou contrôlés avec randomisation, présentent un profil de sécurité ou d'efficacité préliminaire encourageant la poursuite de leur évaluation lors de la prochaine résurgence épidémique. Ces enjeux sont portés aux scénarios à construire et anticiper au cours de la période inter-épidémique inaugurée en 2016. <

En octobre 2016, la publication dans le New England Journal of Medicine des résultats de l'essai international PREVAIL II (partnership for research on Ebola virus in Liberia II) attestait de l'investissement, de la mobilisation sans précédent, et des progrès réalisés dans l'évaluation inédite de plusieurs traitements expérimentaux de la maladie épidémique à virus Ebola ( $E B O V$, pour Ebola virus) [1].

L'épidémie de maladie à virus $\varepsilon$ bola (MVE) qui a sévi en Afrique de l'ouest de 2014 à 2016, a été la plus étendue, vulnérante et difficile à contrôler, depuis la découverte des filovirus ${ }^{1}$. Au cours de cette crise sanitaire sans précédent, plus de 28000 personnes ont été infectées et, parmi ces patients, plus de 11000 sont décédés de MVE aiguë $[2,3](\rightarrow)$.

$\rightarrow$ Voir la Nouvelle de D. Fassin, $m / s n^{\circ} 5$, mai 2015, page 463

\section{Intégrer la \\ recherche clinique \\ dans la réponse \\ aux épidémies}

\section{Les leçons de l'expérience d'Ebola}

Denis Malvy, Daouda Sissoko, Alseny-Modet Camara

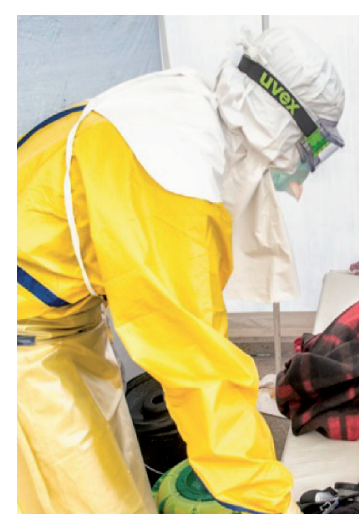

Inserm U1219, université de Bordeaux et centre hospitalouniversitaire de Bordeaux, 46, rue Léo-Saignat, 33076 Bordeaux Cedex, France. denis.malvy@chu-bordeaux.fr

En l'absence de vaccin ou de traitement spécifique homologué, et dès lors que l'état d'urgence de santé publique de portée internationale fut déclaré, la communauté scientifique internationale s'est mobilisée afin d'évaluer le bénéfice de médicaments candidats prometteurs. Cette mobilisation était portée par une obligation morale. Mais elle avait également pour perspective la production de données permettant d'évaluer l'intérêt d'agents antiviraux ou modulateurs de la réponse de l'hôte consécutive à l'infection. Même déployée avec retard, la recherche clinique, concomitamment intégrée au soin, aura compté comme une composante à part entière et emblématique des mesures constitutives de la réponse globale face à l'épidémie. En août 2014, l'Organisation mondiale de la santé (OMS) convoqua une consultation d'experts en éthique sur le bien-fondé de l'évaluation par des essais cliniques à des traitements expérimentaux et de leurs recours pour la MVE épidémique [4]. Ce premier point fut complété en septembre 2014 par la réunion d'experts et de différentes parties prenantes, afin de statuer sur la priorisation des agents à évaluer ${ }^{2}$. Cette priorisation était fondée sur la disponibilité de résultats encourageants obtenus à partir de données établies in vitro et dans des modèles animaux, en l'absence de données concernant les agents testés qui étaient non homologués chez l'homme, dans l'indication de la MVE [5]. Parmi ces candidats, figuraient des petites molécules présentant des activités antivirales directes et des produits de santé d'immunothérapie passive.

${ }^{2}$ www.who.int/entity/csr/resources/publications/ebola/ebola-therapies/en/
${ }^{1}$ Les filovirus, appartiennent à l'ordre des Mononegavirales regroupant les virus à ARN monocaténaire non segmenté à polarité négative. Ils présentent une apparence filamenteuse. 


\section{Immunothérapie passive et stratégies thérapeutiques expérimentales de la maladie à virus Ebola}

S'agissant de l'immunothérapie passive, deux approches principales ont été appliquées: la transfusion de plasma provenant de patients convalescents ayant survécu à la MVE aiguë (comprenant des anticorps a priori neutralisants développés par le patient à la suite de son infection), et le développement, comme équivalents thérapeutiques (de ceux produits naturellement lors de l'infection contrôlée par les patients infectés et convalescents), de combinaisons d'anticorps monoclonaux $(\mathrm{Acm})$ neutralisants ciblant la glycoprotéine d'enveloppe d'EBOV [6].

L'approche représentée par le plasma de convalescents avait l'avantage d'un déploiement rapide. Elle nécessitait néanmoins l'engagement de la population des pays affectés, qui comptait plusieurs milliers de survivants de l'épidémie. Les résultats de son évaluation sur le terrain épidémique en Guinée étaient cohérents avec les données issues d'études réalisées chez le primate non humain avec du sang total de convalescent [7], et n'ont pas mis en évidence de bénéfice en termes de survie. II faut noter toutefois que le titre en anticorps neutralisants spécifiques des culots de plasma, administrés dans le cadre de l'essai conduit chez l'homme, était inconnu au moment de l'administration aux patients enrôlés [8].

Les données préliminaires concernant l'administration d'anticorps monoclonaux dirigés contre la glycoprotéine d'EBOV étaient encourageantes, en particulier pour le ZMapp (Mapp Biopharmaceuticals, San Diego, CA, États-Unis), un cocktail optimisé de trois anticorps présentant une activité neutralisante contre EBOV dans les modèles animaux, avec une efficacité de $100 \%$ sur la survie des primates non humains s'il était administré dans les 5 jours suivant leur infection [9]. Le cocktail ZMapp est la combinaison de trois Acm. II repose, en particulier, sur le développement de deux mélanges d'Acm humanisés $\left(Z M A b^{3}\right.$ et MB-0034). Chacun des trois Acm qui constituent ZMapp (c13C6, c2G4 et $\mathrm{c} 4 \mathrm{G} 7$ ) est dirigé contre un épitope différent de la glycoprotéine de surface du virus Ebola. ZMapp a été initialement développé contre l'espèce EBOV Zaïre-variant Kikwit ${ }^{5}$, mais son activité a été confirmée sur le variant EBOV Zaïre-Makona, responsable de l'épidémie d'Afrique de l'ouest. Au début de l'épidémie, ZMapp n'était disponible qu'en quantité limitée. II avait été administré chez plusieurs patients (intervenants internationaux évacués), dans le cadre d'un usage compassionnel avec un bénéfice apparent ou déclaré, mais qui restait non confirmé. Aucune donnée établissant la dose optimale à utiliser chez l'homme, ni sa fréquence, n'était alors disponible.

\footnotetext{
${ }^{3}$ ZMAb est composé de trois Acm dirigés contre l'enveloppe virale, développés chez la souris ( $1 \mathrm{H} 3,2 \mathrm{G} 4$, et 4G7). $1 \mathrm{H} 3$ et $4 \mathrm{G} 7$ sont des IgG (immunoglobuline de classe G) d'isotype $2 \mathrm{a}, 2 \mathrm{G} 4$ est une IgG2b. Ils ont été produits par immunisation avec la protéine d'enveloppe du virus exprimée par Nicotiana benthamiana, une plante de la famille du tabac.

${ }^{4}$ MB-003 est composé de trois Acm humanisés (c13C6, c6D, h13F6), dont le développement a permis la production d'une forme afucosylée, exprimant une activité de cytotoxicité cellulaire anticorps-dépendante (ADCC). À partir du mélange MB-003 et de la version humanisée des anticorps composant le ZMAb, la recherche de la combinaison optimale de trois Acm a abouti à la combinaison finale (c13C6, c2G4, c4G7), dénommée ZMapp

${ }^{5}$ Kikwit est la ville principale de la province du Kwilu en République démocratique du Congo.
}

\section{Essais cliniques au cours de l'épidémie d'Ebola de 2013-2016 : exemple et enjeux}

Un enjeu emblématique de controverse scientifique mais aussi éthique, a concerné l'élaboration d'essais cliniques qui soient appropriés pour l'évaluation d'un traitement prometteur bien qu'expérimental, dans un contexte de crise humanitaire sans précédent. Le débat portait sur une évaluation qui serait réalisée par essai contrôlé randomisé sous-tendant un tirage au sort entre groupe traité et groupe témoin, ce dernier ne recevant que l'offre de soins de base (encore dénommée standard de soin de support, en anglais standard of care) représentée par le standard de soin du meilleur niveau possible (et qualifié d'optimisé) [10].

Au moment du pic épidémique, la mise en place d'un essai randomisé avec tirage au sort, considéré comme le plus adapté afin de produire le niveau de preuve le plus élevé, ne s'est pas imposée en première intention pour qualifier l'efficacité d'un médicament expérimental prometteur. Cette posture, pragmatique, était liée au contexte du terrain avec (1) l'absence d'expériences préalables de conduite d'un essai au cours de la MVE, (2) la violence de l'expression épidémiologique, avec un nombre élevé de personnes atteintes qui étaient terrifiées, mais, surtout, qui se présentaient simultanément et par vagues, issues du même village et des mêmes familles, (3) les rumeurs et l'hostilité à l'endroit des humanitaires internationaux qui dirigeaient les centres de traitement EBOLA (CTE), et (4) le risque de refus des patients à la propension au soin lié à la perspective d'un tirage au sort dans leur parcours de soin.

L'essai PREVAIL II, encore décliné sous le terme MCM (Ebola medical countermeasures study), est le dernier des trois grands essais cliniques qui a évalué un produit de santé prometteur dans la prise en charge spécifique de la MVE épidémique [1] (Tableau I). II s'agissait d'un essai multi-sites, comparatif, contrôlé, et randomisé. Sa conception comprenait une composante adaptative illustrée par une approche de type bayésien ${ }^{6}$ offrant une part de flexibilité dans la conduite de l'essai [11]. L'approche de type bayésien représentait un compromis qui, tout en comprenant une randomisation, permettait l'interruption précoce et exceptionnelle de l'essai si l'un des traitements expérimentaux se révélait rapidement associé à un niveau d'efficacité dépassant largement celui qui avait été anticipé. Cette approche paraissait bien adaptée au contexte de la MVE épidémique. II s'agissait, en effet, d'une maladie avec un taux de mortalité élevé.

${ }^{6}$ L'approche bayésienne est une méthode statistique permettant de déduire la probabilité de survenue d'un événement à partir de celle d'événements déjà évalués. 
Le but était donc de rendre tout médicament validé disponible dans le délai le plus court possible et pour le plus grand nombre. La survenue de 6 décès (parmi 6 patients) dans le bras recevant le standard de soin de support en comparaison d'aucun décès (parmi 6 patients) dans le groupe traité par le médicament candidat était jugée ainsi suffisante pour interrompre l'essai et valider le médicament.

L'essai PREVAIL II a évalué, sur le terrain épidémique, le cocktail d'anticorps neutralisants ZMapp, administré par voie parentérale [1]. Il a été initié au cours du deuxième trimestre 2015 au Libéria, en Sierra Leone et en Guinée côtière, en période de fin d'expression épidémique. Le contexte social était alors plus favorable compte tenu du contrôle de l'épidémie et de la diminution globale de la mortalité, en partie grâce à l'amélioration de la prise en charge du standard de soin de support dans les CTE. Ces éléments ont indéniablement contribué à l'adhésion des populations aux interventions de santé publique et de déploiement des essais, de même que l'intensification des mesures de contrôle (surveillance active avec micro-cerclage des foyers résiduels, essai pionnier vaccinal « Ebola-ça-suffit $»^{7}$ en Guinée côtière [12]) accompagnées par une mobilisation communautaire. Les participants à l'essai PREVAIL II étaient randomisés de manière équilibrée entre deux bras: le standard de soin de support en cours optimisé (défini comme, au minimum, l'administration intensive des solutés par voie intraveineuse et la surveillance biochimique des électrolytes sanguins); et les mêmes soins, complétés par l'administration de trois perfusions intraveineuses de ZMapp (à la posologie de $50 \mathrm{mg} / \mathrm{kg}$ tous les trois jours). En Guinée, le standard de soin de support en cours comprenait l'administration du favipiravir (Toyama Chemical, Tokyo, Japon), un antiviral repositionné dans l'indication de la MVE et administré par voie orale. Le favipiravir avait été évalué dans un essai comparatif non randomisé avec témoins historiques, entre décembre 2014 et février 2015, déployé en Guinée forestière, au moment du pic de l'épidémie (Tableau l et Figure 1). Les résultats encourageants qui avaient été obtenus en vue de la poursuite de son évaluation avaient convaincu les autorités nationales de mettre en place un programme de prescription encadrée dans les CTE de Guinée [13]. Soixante-douze patients (sur l'effectif ciblé de 200 personnes, non atteint compte tenu de la résolution de l'épidémie) ont été inclus dans l'essai PREVAIL II, et stratifiés en fonction du lieu de leur recrutement et de leur charge virale inaugurale. La charge virale du virus Ebola au moment de I'inclusion, était quantifiée par RT-PCR (real time-reverse transcription polymerase chain reaction) quantitative et exprimée en nombre de cycles $(\mathrm{Ct})$ nécessaires pour atteindre le seuil choisi représentant le nombre de copies de virus dans le sang. Le ZMapp n'a pas prouvé un bénéfice statistiquement significatif sur la mortalité. Cependant, une tendance favorable, suggérée par les $37 \%$ de mortalité dans le bras témoin au regard des $22 \%$ dans le bras intervention, a pu être observée. L'effectif de l'échantillon restant faible compte tenu de la limitation du recrutement des participants, ce résultat n'est pas surprenant. II correspondait cependant à une diminution de $40 \%$

${ }^{7}$ L'essai clinique du vaccin rVSV-Ebov, baptisé « $\varepsilon$ bola ça suffit » en Guinée, s'est déroulé entre avril et juillet 2015 et a impliqué 7500 personnes. du risque relatif de décès avec traitement par ZMapp (risque relatif bayésien : 0,62 ; intervalle de confiance : $[0,29-1,24])$. Le standard de soin de support, incluant le favipiravir en Guinée, était associé, d'autre part, à une tendance favorable dans les analyses stratifiées en sous-groupes selon le site, même si le nombre limité de patients inclus était trop faible pour conclure.

À l'avenir, les travaux qui seront conduits pour valider les modes d'administration et les posologies des protocoles devront compter avec la conduite d'une évaluation thérapeutique réalisée dans des situations sanitaires extrêmes, à savoir son inclusion dans un projet de soin dont l'organisation devra composer avec le paysage, toujours inconnu, tributaire des épidémies suivantes [14]. Ainsi, les contingences logistiques techniques et humaines, en rapport avec l'administration d'un traitement de ce type, restent une contrainte en contexte de zones reculées et isolées. Outre la nécessité d'une chaîne du froid constante pour le transport et la conservation du produit, son administration impose une surveillance continue et attentive de plusieurs heures, à intégrer au roulement des équipes de soins, dans le mode opérationnel actuel de centres de traitement.

\section{La recherche clinique à l'heure inter-épidémique}

En 2017, les stratégies thérapeutiques prometteuses ayant fait l'objet d'une évaluation ne se sont pas avérées définitivement supérieures au seul standard de soin de support $[1,8,13]$ (Tableau 1). Mais un point d'étape est apparu pour la priorisation des futures évaluations per-épidémiques et la conception de modèles animaux prédictifs et pertinents. Certaines molécules ou classes médicamenteuses, initialement prometteuses, ont en effet été disqualifiées $[10,15,16]$. Néanmoins, les données de tolérance et d'efficacité préliminaires de certains agents encouragent la poursuite de leur évaluation, en particulier dans le cadre de combinaisons thérapeutiques (Tableau I) [10, 17$]$. II s'agit, en particulier, du ZMapp [1] et du favipiravir. L'évaluation du bénéfice de l'antiviral favipiravir nécessitera le recours à des doses plus élevées que celles qui ont été utilisées dans l'essai clinique pionnier $[13,18]$. Un troisième agent également prometteur, le GS 5734 (Gilead Sciences, Foster City, CA, États-Unis), est en cours d'investigation. II s'agit d'un analogue nucléotidique, administré par voie parentérale qui a été utilisé, à titre compassionnel, au cours de la MVE aiguë. À l'échéance d'août 2017, il faisait l'objet d'un essai toujours ouvert au Libéria dans la MVE chronique chez le survivant masculin avec portage du virus dans le tractus génital (Tableau I) [17]. 


\begin{tabular}{|c|c|c|c|c|}
\hline Agent & $\begin{array}{l}\text { Laboratoire } \\
\text { pharmaceu- } \\
\text { tique } \\
\text { (localisation) }\end{array}$ & Description & $\begin{array}{l}\text { Essai (s) clinique (s) } \\
\text { Promoteur } \\
\text { Statut (octobre 2017) }\end{array}$ & Schéma de l'étude \\
\hline $\begin{array}{l}\text { Favipiravir } \\
\text { (T-705; } \\
\text { Avigan) } \\
{[10,13]}\end{array}$ & $\begin{array}{l}\text { Toyama Chemical, } \\
\text { Fujifilm group } \\
\text { (Tokyo, Japon) }\end{array}$ & $\begin{array}{l}\text { 6-fluoro-3-hydroxy-2-pyrazinecar- } \\
\text { boxamide } \\
\text { Homologué dans le traitement de } \\
\text { l'infection par le virus de la grippe } \\
\text { (Avigan) au Japon, mars } 2014 \\
\text { Inhibiteur de la (L) polymérase de } \\
\text { I'ARN viral }\end{array}$ & $\begin{array}{l}\text { JIKI } \\
\text { Initié en décembre 2014, Guinée } \\
\text { MVE aiguë (NCT02329054) } \\
\text { Inserm, France } \\
\text { Terminé } \\
\text { FORCE } \\
\text { Initié en avril 2016, Guinée } \\
\text { MVE chronique (persistance de produc- } \\
\text { tion virale dans le réservoir génital mas- } \\
\text { culin) (NCT02739477) } \\
\text { Inserm, France } \\
\text { En cours }\end{array}$ & $\begin{array}{l}\text { Phase II-Essai multicentrique } \\
\text { de preuve de concept, témoins } \\
\text { historiques, analyses séquen- } \\
\text { tielles }\end{array}$ \\
\hline $\begin{array}{l}\text { Plasma } \\
\text { de convales- } \\
\text { cents } \\
\text { [8] }\end{array}$ & Sans objet & $\begin{array}{l}\text { Plasma ABO-compatible de donneurs } \\
\text { convalescents (pas de vérification du } \\
\text { taux d'anticorps neutralisants) }\end{array}$ & $\begin{array}{l}\text { Dénommé Ebola-Tx } \\
\text { Initié en février 2015, Guinée } \\
\text { MVE aiguë (NCT02342171) } \\
\text { ITM, Belgique } \\
\text { Terminé }\end{array}$ & $\begin{array}{l}\text { Phase II/III-Essai non rando- } \\
\text { misé, témoins historiques }\end{array}$ \\
\hline $\begin{array}{l}\text { Zmapp } \\
{[1]}\end{array}$ & $\begin{array}{l}\text { MappBio (San Diego, } \\
\text { CA, États-Unis) }\end{array}$ & $\begin{array}{l}\text { Cocktail des trois anticorps neutra- } \\
\text { lisants humanisés c13C6, } 2 \text { G4 et 4G7 } \\
\text { sélectionnés à partir des cocktails } \\
\text { d'anticorps MB-003 et ZMab } \\
\text { Cible la glycoprotéine virale }\end{array}$ & $\begin{array}{l}\text { PREVAIL II } \\
\text { Initié en février 2015, international } \\
\text { MVE aiguë (NCT02363322) } \\
\text { NIAID, États-Unis et Inserm, France } \\
\text { En cours }\end{array}$ & $\begin{array}{l}\text { Phase I/II -Essai comparatif } \\
\text { randomisé multicentrique, } \\
\text { schéma adaptatif; compa- } \\
\text { raison avec le SOC optimisé } \\
\text { seul (incluant le favipiravir en } \\
\text { Guinée) }\end{array}$ \\
\hline $\begin{array}{l}\text { TKM-130803 } \\
\text { (TKM) } \\
{[15]}\end{array}$ & $\begin{array}{l}\text { Arbutus Biopharma } \\
\text { (anciennement } \\
\text { Tekmira Pharmaceu- } \\
\text { ticals), Burnaby, } \\
\text { Canada }\end{array}$ & $\begin{array}{l}\text { Petite molécule ARN-intercalante } \\
\text { Cible les ARN messagers de la (L) } \\
\text { polymérase de l'ARN viral et de la pro- } \\
\text { téine } 35 \text { (VP35) }\end{array}$ & $\begin{array}{l}\text { RAPIDE-TKM } \\
\text { Initié en Février 2015, Sierra Leone } \\
\text { MVE aiguë (PACTR201501000997429) } \\
\text { Université d'Oxford, Royaume Uni } \\
\text { Interrompu }\end{array}$ & $\begin{array}{l}\text { Phase II-essai mono-bras, } \\
\text { témoins historiques et concur- } \\
\text { rents, approche multi-états; } \\
\text { associé à une étude simultanée } \\
\text { d'observation }\end{array}$ \\
\hline $\begin{array}{l}\text { Brincidofovir } \\
\text { (CMX001) } \\
{[16]}\end{array}$ & $\begin{array}{l}\text { Chimerix } \\
\text { (Durham, NC, } \\
\text { États-Unis) }\end{array}$ & $\begin{array}{l}\text { Analogue nucléotidique conjugué lipi- } \\
\text { dique, prodrogue du cidofovir } \\
\text { Mode d'action sur le virus Ébola non } \\
\text { connu } \\
\text { Activité antivirale ne nécessitant pas } \\
\text { de phosphorylation }\end{array}$ & $\begin{array}{l}\text { RAPIDE-BCV } \\
\text { Initié en Octobre 2014, Liberia } \\
\text { MVE aiguë (NCT02271347) } \\
\text { Université d'Oxford, Royaume Uni } \\
\text { Inabouti }\end{array}$ & $\begin{array}{l}\text { Phase II-essai mono-bras } \\
\text { témoins historiques et concur- } \\
\text { rents, approche multi-états }\end{array}$ \\
\hline GS-5734 & $\begin{array}{l}\text { Gilead (Foster City, } \\
\text { CA, États-Unis) }\end{array}$ & $\begin{array}{l}\text { Prodrogue d'un analogue nucléoti- } \\
\text { dique de l'adénosine } \\
\text { Inhibiteur de la polymérase (L) de } \\
\text { I'ARN viral }\end{array}$ & $\begin{array}{l}\text { PREVAIL } 4 \\
\text { Initié en Juin 2016, Libéria } \\
\text { MVE chronique (persistance de produc- } \\
\text { tion virale dans le réservoir génital mas- } \\
\text { culin) (NCT02818582) } \\
\text { NIAID, États-Unis } \\
\text { En cours }\end{array}$ & $\begin{array}{l}\text { Phase II- Essai comparatif ran- } \\
\text { domisé, double-bras, double- } \\
\text { insu, versus placebo }\end{array}$ \\
\hline
\end{tabular}

Tableau I. État des lieux des principaux essais thérapeutiques des traitements expérimentaux spécifiques de la maladie épidémique à virus Ebola. Ct : nombre de cycles; ITM : Institute of Tropical Medicine ; J : jour ; MVE : maladie à virus Ébola ; NIAID : National institute of allergy and infectious diseases ; SOC : standard de soins de support ; IC : intervalle de confiance (adapté de [10]). 


\section{Formulation-Posologie}

Orale

Dose de charge de 6 g à $\mathrm{Jl}$, complété par une dose d'entretien de $2,4 \mathrm{~g} / \mathrm{j}$ en deux prises de J2 à $J 10$

\section{Résultats}

Parmi 99 participants adultes, mortalité de $20 \%$ (IC $95 \%, 11,6 \%-32,4 \%$ ) chez ceux avec $\mathrm{Ct} \geq 20$ et de $91 \%$ (IC $95 \%$, $78,8 \%-91,1 \%$ ) chez ceux avec $\mathrm{Ct}<20$ Valeurs résiduelles basses au cours de la phase d'entretien

\section{Commentaires}

Mortalité non significativement différente avec la valeur-cible prédéfinie de $30 \%$ pour les patients avec valeur de Ct élevée et de $85 \%$ pour les patients avec valeur de Ct basse

Signal d'efficacité préliminaire chez le patient avec charge virale modérément élevée

Absence de bénéfice de la monothérapie à la dose de l'essai chez le patient avec charge virale très élevée [13]

Doses plus élevées à évaluer [18]
Transfusion intra-veineuse

2 transfusions de $200-250 \mathrm{ml}$ de plasma, avec chaque unité de plasma obtenue d'un donneur différent, administrée à moins de 2 jours suivant le diagnostic

Injection intraveineuse

$50 \mathrm{mg} / \mathrm{kg}$ dans les $24 \mathrm{~h}$ suivant l'inclusion, complétée par 2 doses supplémentaires à 3 jours d'intervalle

Injection intraveineuse $0,3 \mathrm{mg} / \mathrm{kg}$ une fois par jour, 7 jours

Orale

Dose de charge de $200 \mathrm{mg}$ à $\mathrm{Jl}$, complété par une dose d'entretien de $100 \mathrm{mg}$ à $\mathrm{J4}, \mathrm{J} 8$, J11, et J15
Parmi 84 participants, mortalité à J3-J16 de $31 \%$ dans le groupe plasma de convalescents et de $38 \%$ dans le groupe témoin (odds ratio, 0,88 - IC $95 \%$, 0,51-1,51), ajusté sur la Ct et l'âge Effectif non atteint (72 participants sur les 200 attendus)

Mortalité à 28 de $37 \%(13 / 35)$ dans le groupe SOC versus $22 \%(8 / 36)$ dans le groupe SOC+ZMapp

Mortalité parmi ceux avec charge virale très élevée $(\mathrm{Ct} \leq 22)$ à l'admission de $60 \%$ (9/15) dans le groupe SOC versus $47 \%$ (7/45) dans le groupe SOC+ZMapp Survie à 14 après le début de la MVE de $25 \%(3 / 12)$, après exclusion de 2 décès précoces dans les 48 premières heures suivant l'inclusion

Essai interrompu après analyse de futilité (survie à $\mathrm{Jl} \leq 55 \%$ )

Recrutement interrompu après 4 inclusions. Survie à $\mathrm{J} 14$ après le début de la MVE de 0/4 patients
Absence de bénéfice sur la mortalité en comparaison avec les témoins historiques

Absence de bénéfice significatif sur la survie, mais manque de puissance

Contrainte liée à l'administration (2-12 h) et aux effets indésirables (réactions systémiques) nécessitant une pré-médication Spécifique au virus Ebola-Zaïre, comprenant la souche Makona (épidémique)

Absence de bénéfice apparent, possible facteur de confusion dû à l'inclusion de patients en stade avancé de la MVE Effets indésirables systémiques (syndrome de production cytokinique aiguë) chez le volontaire sain Agent disqualifié

Pré-requis de concept d'activité improbable Agent disqualifié

Injection intraveineuse

$100 \mathrm{mg}$ de GS-5734 ou placebo une fois par Non disponible Candidat prometteur 


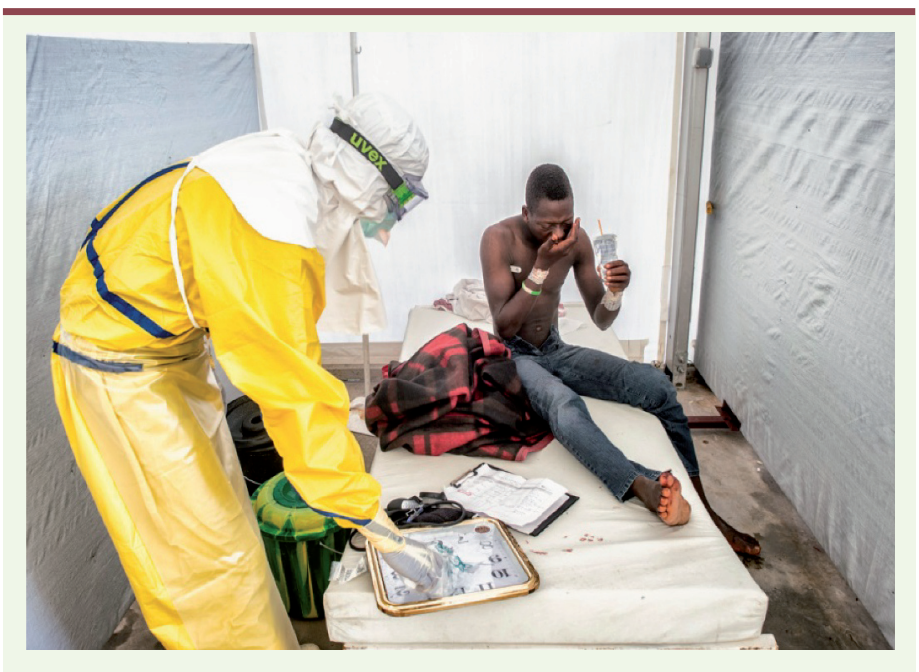

Figure 1. Prise en charge d'un patient atteint de maladie à virus Ebola. Essai JIKI, Centre de traitement Ebola, Nzérékoré, Guinée, 2015 (Photo (c) Sylvain Cherkaoui/Cosmos pour ALIMA [13]).

À la suite de son évaluation per-épidémique, le ZMapp fait l'objet d'une procédure internationale (dans les trois pays d'Afrique de l'ouest frappés par la MVE épidémique de 2013-2016) d'usage étendu (expanded use protocol) visée par la Food and drug administration (FDA). Le favipiravir reste, en 2017, toujours dispensable dans le cadre d'un programme de prescription encadrée (équivalent d'une autorisation temporaire d'utilisation de cohorte) en Guinée [19]. Ce cadre est en tout point convergent avec celui défini par l'OMS sous l'acronyme anglais MEURI (pour monitored emergency use of unregistered and experimental interventions) [4, 20]. Le MEURI correspond à I'utilisation et à l'évaluation de médicaments expérimentaux au cours des épidémies à venir, en l'absence de la possibilité d'essais de recherche clinique qui puissent être déployés. Parallèlement aux promesses qu'apportent les développements de vaccins spécifiques du virus, l'identification et l'homologation de traitements spécifiques de la MVE restent cependant un enjeu important. Elles s'inscrivent autant dans la prise en charge des cas de MVE aiguë, que dans le contexte des manifestations cliniques comme le syndrome post-Ebola ${ }^{8}$, de la persistance du virus dans le sperme des sujets masculins ayant survécu, et le risque de résurgence de foyers épidémiques retardée $[10,19,21]$.

En période inter-épidémique, plusieurs axes de recherche sont développés en termes d'anticipation et de prospective. Le premier axe est porté par la recherche expérimentale, en particulier chez le

\footnotetext{
${ }^{8}$ Le syndrome du survivant de MVE (ou syndrome post- bbola) rend compte des complications tardives et séquelles exprimées à l'issue de la phase résolutive de la MVE aiguë. Les manifestations cliniques les plus fréquentes regroupent troubles musculo-squelettiques (poly-arthralgies chroniques), perte de la vision (uvéite, cataracte) et troubles neuro-cognitifs. Leur mécanisme est présumé d'origine post-infectieuse, réactionnelle ou auto-immune. Néanmoins, la contribution de la persistance et de la réplication virale au sein de sanctuaires anatomiques ou immunologiques préservés (système nerveux central, tractus génital masculin) a été établie. II constitue, avec les rares cas repérés de recrudescence tardive de MVE (panuvéite, méningo-encéphalite) et la persistance virale prolongée dans les fluides corporels (liquide séminal), le cadre de la MVE chronique.
}

primate non humain. II a comme objectifs d'identifier de nouveaux modes d'action contre le virus qui permettraient de viser plusieurs cibles, d'adapter la posologie d'agents qui se sont révélés prometteurs et de concevoir des modèles pertinents afin d'évaluer des combinaisons sur le terrain épidémique.

Le second enjeu à relever renvoie au développement et à l'adaptation d'essais cliniques innovants qui soient éthiquement acceptables, performants et adaptés aux crises épidémiques. Dans cette perspective, il faut compter avec des probabilités et des inconnues [22]. Les prochaines émergences de MVE, ou d'autres fièvres virales hémorragiques, concerneront des populations vulnérables, isolées, frappées par la pauvreté, avec des scénarios déterminés par des facteurs contextuels psycho-sociaux, géopolitiques, économiques et sanitaires. Nonobstant l'absence de modèle épidémiologique prédictif, la structuration, en cours, des mécanismes permettant le renforcement des systèmes de santé locaux et de surveillance épidémiologique inter-régionaux devrait permettre de prévenir de voir se renouveler l'expression de flambées expansives et catastrophiques que l'on a connues lors de la crise sanitaire de 20132016.

Un exemple nous en est fourni, avec l'expression épidémique du foyer d'Ebola, qui a marqué la fin de la période post-épidémique inaugurée en 2016 et qui est survenue au cours des mois d'avril à mai 2017 dans la région du bas-Uélé en République démocratique du Congo. Le phénomène était caractérisé par une expression d'Ebola de type historique, dans une région extrêmement reculée. Son investigation aura rapporté, deux cas confirmés, trois probables et 12 suspects. En regard de cette évidence, l'approche adaptative de recherche de preuve appliquée dans l'essai PREVAIL II prévoyait un effectif de 200 participants (en terme de puissance), ce qui, même dans un scénario d'anticipation et de réactivité de mise en place précoce d'un essai, ne devrait pas être atteint. Les stratégies d'innovation méthodologique dans la recherche de preuve devront composer avec l'expression de cas groupés d'effectifs qui ne dépasseront pas quelques dizaines, et qui se répètera de manière plus ou moins étalée dans le temps. Cette situation impose de disposer d'un maillage géographique, avec déploiement auprès des communautés, de sites de diagnostic (disposant de tests rapides), de prise en charge et d'investigation clinique. Ce dispositif opérationnel est la condition d'un recrutement de patients qui soit le plus précoce possible, corolaire à la fois de leur participation, afin de bénéficier des traitements expérimentaux de la maladie, et, le cas échéant, du contrôle et de l'homogénéité dans le 
temps, de l'efficacité du traitement, au cours de l'essai. Le nombre de stratégies prometteuses candidates à évaluation chez l'homme sera inévitablement limité, que ces stratégies renvoient à une administration en monothérapie comme en combinaison, ou qu'elles soient issues de la génération de classes thérapeutiques nouvellement développées, c'est-à-dire hors de produits déjà disponibles comme le favipiravir, le ZMapp ou le GS 5734. Enfin, les stratégies de recherche clinique devront compter avec une adaptation au contexte : l'optimisation du support de prise en charge du patient et de gestion des défaillances systémiques constitutives de la MVE; l'impact d'une évolution des mesures barrières d'équipement de protection personnelle; ou la disponibilité d'un vaccin efficace pour les professionnels de santé.

\section{Conclusion}

Au cours de la période inter-épidémique, les avancées sur les enjeux et défis à relever dans le contexte de la MVE épidémique sont autant de repères attendus dans l'objectif d'explorer le bénéfice de molécules au cours des formes aiguës et chroniques de telles maladies infectieuses ayant pour origine des agents hautement pathogènes, qui frappent des populations vulnérables en particulier d'Afrique sub-sahélienne, et qui ont la capacité de s'étendre ou de s'exporter dans un monde globalisé. $\diamond$

\section{SUMMARY}

Integrating clinical research into epidemic response: the field perspective in the Ebola experience

During the 2013-2016 west African Ebola outbreak that affected West Africa, accelerated clinical trials, testing unproven but promising and potentially lifesaving experimental interventions emerged as a key component of the global outbreak. In 2017, no Ebola medical countermeasures had proven antiviral efficacy in patients. However, in September 2014, the World Health Organization inventoried a list of potential drug candidates developed or repurposed with demonstrated antiviral efficacy in vitro or in animal models. Numerous therapeutics were considered or explored during the outbreak, including nucleoside and nucleotide analogues, nucleic acid-based drugs and immunotherapeutics. Drugs in clinical trials were tested within the framework of optimized supportive care with fluids and electrolytes and management of severe compromise of multiple organs resulting from viral cytopathology and immune-mediated cell damage. Assessment of those therapeutics with encouraging preliminary efficacy or safety profile, like the repurposed direct antiviral agent favipiravir or the combination of antibodies ZMapp requires further investigation to confirm their efficacy in humans, propose appropriate doses and evaluate the possibility of treatment combinations. During the lull before the next epidemic, major challenges for managing future Ebola epidemics include scientific, clinical and public health preparedness with establishment of innovative patient care and clinical research support in remote poor areas where Ebola and other deadly infectious diseases typically reemerge. $\diamond$

\section{LIENS D'INTÉRÊT}

Les auteurs déclarent n'avoir aucun lien d'intérêt concernant les données publiées dans cet article.

\section{RÉFÉRENCES}

1. PREVAIL II writing group; Multi-national PREVAIL II study team, Davey RT $\mathrm{Jr}$, Dodd L, Proschan MA, et al. A randomized, controlled trial of ZMapp for Ebola virus infection. $N$ Engl J Med 2016 ; 375 : 1448-56.

2. Fassin D. Santé globale, un nouveau concept ? Quelques enseignements de l'épidémie à virus Ébola. Med Sci (Paris) 2015 ; 31 : 463-4.

3. Briand $S$, Bertherat $\varepsilon$, Cox P, et al. The international Ebola emergency. New Engl J Med 2014 ; 371:1180-3.

4. Krech R, Kieny MP. The 2014 Ebola outbreak: ethical use of unregistered interventions. Bull World Health Organ 2014 ; 92: 622.

5. Yazdanpanah Y, Arribas JR, Malvy D. Treatment of Ebola virus disease. Intensive Care Med $2015 ; 41: 115-7$.

6. Zeitlin L, Whaley KJ, Olinger GG, et al. Antibody therapeutics for Ebola virus disease. Curr Opin Virol 2016; $17: 45-9$.

7. Jahrling P, Geisbert T, Geisbert J, et al. Evaluation of immune globulin and recombinant interferon- $\alpha 2 b$ for treatment of experimental $\varepsilon$ bola virus infections. J Infect Dis 1999 ; 179 (suppl 1) : S224-34.

8. van Griensven J, Edwards T, de Lamballerie X, et al. Evaluation of convalescent plasma for Ebola virus disease in Guinea. N Engl J Med 2016 ; $374: 33-42$.

9. Qiu X, Wong G, Audet J, et al. Reversion of advanced Ebola virus disease in non-human primates with ZMapp. Nature $2014 ; 514: 47-53$.

10. Malvy D. Science in a crisis. Medical countermeasures in Ebola virus disease, 2016: lessons learned and perspectives. Bull Soc Pathol Exot 2016; 109 : 262-71

11. Dodd LE, Proschan MA, Neuhaus J, et al. Design of a randomized controlled trial for Ebola virus disease medical countermeasures: PREVAIL II, the Ebola MCM Study. J Infect Dis 2016 ; 213 : 1906-13.

12. Henao-Restrepo AM, Camacho A, Longini IM, et al. Efficacy and effectiveness of an rVSV-vectored vaccine in preventing Ebola virus disease: final results from the Guinea ring vaccination, open-label, clusterrandomised trial (Ebola ça suffit !). Lancet 2017 ; 389 : 505-18.

13. Sissoko D, Laouénan C, Folkesson $\varepsilon$, et al. Experimental treatment of favipiravir for Ebola virus disease (the JIKI trial): a historically-controlled, single arm proof-of concept trial in Guinea. PLoS Med 2016; 13 : e1001967.

14. Duraffour S, Malvy D, Sissoko D. How to treat Ebola virus infections? A lesson from the field. Curr Opin Virol 2017 ; 24 : 9-15.

15. Dunning J, Sahr F, Rojek A, et al. Experimental treatment of Ebola virus disease with TKM-130803: A single-arm Phase 2 clinical trial. PLoS Med 2016 ; 13 : el001997.

16. Dunning J, Kennedy SB, Antierens A, et al. Experimental treatment of Ebola virus disease with brincidofovir. PLoS One 2016; 11:e0162199.

17. Warren TK, Jordan R, Lo MK, , et al. Therapeutic efficacy of the small molecule GS-5734 against Ebola virus in rhesus monkeys. Nature 2016; 531 : 381-5.

18. Nguyen TH, Guedj J, Anglaret X, et al. Favipiravir pharmacokinetics in Ebola-Infected patients of the JIKI trial reveals concentrations lower than targeted. PLoS Negl Trop Dis 2017 ; 11 : e0005389.

19. Sissoko D, Duraffour $S$, Kerber R, et al. Persistence and clearance of Ebola virus RNA from seminal fluid of Ebola virus disease survivors: a longitudinal analysis and modelling study. Lancet Glob Health 2017 ; 5 : e80-e88.

20. Ethical issues related to study design for trials on therapeutics for Ebola Virus Disease. 2014. Report of the WHO ethics working group meeting. 20-2 October 2014. Geneva : World health Organization,2014.http://apps.who. int/iris/bitstream/10665/137509/1/WHO_HIS_KER_GHE_14.2_eng.pdf

21. Diallo B, Sissoko D, Loman NJ, et al. Resurgence of Ebola virus disease in Guinea linked to a survivor with virus persistence in seminal fluid for more than 500 days. Clin Infect Dis 2016 ; 63 : 1353-6.

22. Maehira $Y$, Kurosaki $Y$, Saito $T$, et al. Responding to ever-changing epidemiological dynamics of Ebola virus disease. BMJ Global Health 2016 ; $1: \mathrm{e} 000180$. 